\title{
Defect Analysis and Innovation Design of Synchronizer for Clutchless Automatic Mechanical Transmission
}

\author{
Lipeng Zhang ${ }^{1}$, Yunao Peng ${ }^{1}$, Haojie Yang ${ }^{1}$ and Shaohua $\mathrm{Li}^{2^{*}}$
}

\begin{abstract}
The synchronizer is a key component of automatic mechanical transmission (AMT) equipped in electric vehicles, but the inertial lock-ring synchronizer (ILRS) commonly used there is not suitable especially for pure electric vehicles without a clutch because of big shift impact. To make the shifting process rapid and smooth, a new synchronizer named pressure-controllable friction ring synchronizer (PCFRS) was designed. Initially, the inevitable shortcoming of ILRS was verified by simulation and test. Furthermore, the mechanical characteristics and advantages of the new synchronizer over ILRS were analyzed. Then, the formulations describing the dynamic transmission based on the working mechanism of the PCFRS were established. Finally, the shifting simulation results with PCFRS and ILRS based on the same operating conditions were compared and analyzed. The research shows that the PCFRS can meet the main shifting evaluation index of an AMT without complex control methods, as well as it takes only $0.2406 \mathrm{~s}$ to finish the comfortable and zero-speed-difference shifting. The shifting quality of PCFRS is better than that of the ILRS. It lays a foundation for using the new synchronizer as a part of clutchless AMTs equipped in pure electric vehicles.
\end{abstract}

Keywords: Electric vehicle, Clutchless automatic mechanical transmission, Inertial lock-ring synchronizer, Pressurecontrollable friction ring synchronizer, Mechanical characteristics, Shifting quality

\section{Introduction}

Pure electric vehicles, as crucial parts of new energy vehicles, are the important researching objects in the automotive industry in response to the energy crisis and environmental pollution [1]. The automatic mechanical transmission (AMT) can enhance their power and economy, as well as improve the motor and controller unit's lifespan [2], so it is in vogue to put an AMT in the transmission systems of pure electric vehicles. For the motor of pure electric vehicles, the driving torque and speed can be controlled more easily, so the motor can reduce the need for clutches due to the active speed regulation, and the clutchless AMT is in popular [3, 4]. The shifting

\footnotetext{
*Correspondence: lishaohua@stdu.edu.cn

${ }^{2}$ State Key Laboratory of Mechanical Behavior and System Safety of Traffic Engineering Structures, Shijiazhuang Tiedao University,

Shijiazhuang 050043, China

Full list of author information is available at the end of the article
}

quality of clutchless AMT directly affects the dynamic performance, economy and driving comfort of an electric vehicle [5]. Thus, a synchronizer with better shifting quality is required for the clutchless AMT because of the duty of achieving the speed synchronization between the sleeve and the gear ring [6,7]. To be specific, it can reduce the shifting impact and noise. In addition, it can make the shifting process steady and extend the service lifespan of AMTs [8]. As a core component, the performance has an important influence on the shifting quality, the research on synchronizers is significant.

Different shifting control strategies based on the working mechanism of ILRS are proposed to improve the shifting quality. Shifting force is a key factor affecting shifting quality. Wang et al. [9] adopted a position and force switching control scheme to avoid the problem that the shifting force produces a large impact. For the lock position, as the shifting occurs, the friction coefficient 
between the synchro ring and the friction cone ring will decrease and cause shifting failure [10]. Besides, the rapid adjustment of the motor speed and the rapid teeth feeding of the sleeve make the synchronizer fail to lock teeth. Qin et al. [11] adopted a gear shifting control strategy based on parallel coordinated control to realize reliable, smooth and fast gear shifting. In addition, the input shaft is directly connected with the drive motor, resulting in a greater inertia of the input shaft, therefore a greater impact when the gears are engaged. For the above questions, Lin et al. [12] designed an inverse system and an active disturbance rejection controller to reduce the impact and synchronization time. Zhang et al. [13] proposed a stage-by-phase multivariable combination controller based on the control of position, velocity and force of the actuators, which reduces the impact of mode switching and improves the dynamic performance of electric vehicles. Zhang et al. [14] proposed a synchronizer shifting trajectory tracking controller. Although the above researches have achieved effective results in gear shifting, the control algorithms may be too complex to complete system identification and parameters correction because of the very short shifting time. Besides, due to the randomness of shifting and the wear of mechanical parts, the reliability of the strategies need to be improved.

The structural parameters of the synchronizer have various effects on the synchronization performance, locking performance, shifting quality and service lifespan [15]. Different parts of synchronization also have different degrees of influence [16, 17]. Wang et al. [18] optimized the parameters of synchronizer to effectively shorten the synchronization time and increase the lifespan of the synchronizer. The researchers have effectively improved the shifting quality of the AMT using the inertial lock-ring synchronizers (ILRSs). However, in combination with the characteristics of the ILRS and the electric motor, the following problems still exist. First, the sleeve can not be moved before completing synchronization. Second, there is a break-through load when the sleeve breaks away from the slider [19], which is unpredictable and difficult to control. Next, the ILRS will cause two large impacts during the gear shifting. At last, combined with the shifting process of the ILRS, the synchro ring will not work after the synchronization phase, the speed difference will generate in the final turn-teeth phase, and then it will generate a huge impact [20].

With the application of motors in the new energy vehicles, the clutchless mechanical direct-connection motor-transmission systems are increasingly popular in the electric vehicles. The research on the direct-connection motor-transmission system mainly focuses on the application of various motor-controlling algorithms to achieve the speed synchronization of the sleeve and the gear ring, and the feasibility of using adapter sleeves to shifting gears. Bóka et al. [21] established the dynamic model of the jointing process of the synchroniserless system, and analyzed the influence of the relative speed and relative angle of the jointing sleeve and the jointing gear ring on the gear shifting. Yu et al. [22] studied the control of the synchronization speed during gear engagement and motion control of the gear-change actuator mechanism. The speed synchronization of the sleeve and the target gear is realized. The researchers have made great progress in the speed relationship between the sleeve and the target gear, which is a clue for the development of clutchless AMT. However, the direct shifting method using the sleeve requires high control accuracy and strict conditions, and the control algorithm requires wonderful real-time performance of the drive system, which makes it difficult to use the sleeve for high quality shifting.

Regarding the electromechanical coupling of gear shifting in pure electric vehicles, some scholars have studied novel synchronization mechanisms to replace traditional synchronizers to achieve high quality shifting. A British innovation company-Zero shifting has developed a new system which is replace the synchronizer with the Zero meshing ring mechanism, and using two sliders to achieve shifting without power interruption [23]. However, two sets of shifting forks are needed to realize the shifting, which makes the shifting actuator more complicated and harder to control. Mo et al. [24, 25] proposed a new type of synchronizer called harpoon-shifting, which is replaced the friction cone ring of the ILRS with a torsion spring to eliminate the speed difference. It overcomes the shortcomings of friction synchronous shifting. However, torsion springs greatly limits the range of the shifting speed difference. If the speed difference between the sleeve and the meshing gear ring is too large, the spring will be compressed to the maximum value and cause greater impact; if the speed difference is too small, there may be a speed difference in the gear advancement process. To sum up, the existing new synchronizers are still defective when applied to pure electric vehicles.

In summary, the clutchless AMT of pure electric vehicles using traditional ILRSs is not suitable, the way of directly canceling the synchronizer and adopting the method of sleeve shifting is relatively difficult and requires high-precision sensors, all of them are terrible influencing factors. Therefore, the better shifting scheme is to adapt a suitable synchronizer with shifting control method for easily achieving shifting. In response to the above problems, a pressure-controllable friction ring synchronizer (PCFRS) is proposed in the paper, which realizes synchronization through compression springs and does not require lock teeth of gear during synchronization. There is no slider mechanism, that is, it is not 
necessary for the sleeve to apply the overtaking force that breaks off the constraint of the slider. In the gear-shifting stage, the generated speed difference can be eliminated synchronously to achieve no-speed-difference shifting.

The structure of the paper is as follows. Section 2 provides the shifting simulation and experiment results about the ILRS. Section 3 analysis the working mechanism and shifting strategy of the PCFRS. Section 4 establishes the dynamics model of the meshing. Section 5 simulates gear shifting and analyzes the simulation results about the PCFRS and ILRS. Section 6 draws the conclusion, summarizes and looks forward to this paper.

\section{Simulation and Experiment Analysis of the ILRS}

\subsection{Modelling and Simulation Analysis of ILRS}

The whole structure of ILRS is represented in Figure 1. It is unable to eliminate the speed difference caused by the engagement of the shifting. Therefore, in order to achieve better shifting, the position control of the Proportional Integral Derivative (PID) is used to realize the shifting force control with different $P$ values. Among them, the $P$ value is a proportional reflection of the deviation signal of the control system. Once the deviation occurs, the controller immediately produces control effect to reduce the deviation. The $P$ parameter quickly acts on the output. The larger the $P$ value is, the stronger the effect will be, and the dynamic response will be faster. Different shifting modes are used for multiple sets of simulations, which are designed to simply and intuitively reflect the mechanical characteristics of the ILRS. The goal is to make it comparable to the corresponding mechanical characteristics of the PCFRS.

The shifting process of the ILRS is shown in Figure 2, which shows the relationship between the input shaft speed and the output shaft speed and the displacement of the sleeve at all stages. Combined with the simulated speed curve and displacement curve, point $A$ indicates that the ILRS completes the pre-synchronization phase

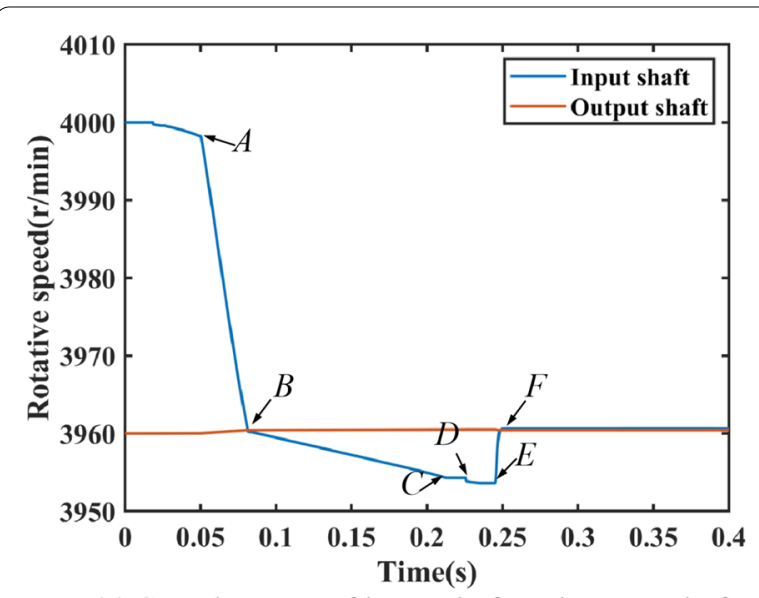

(a) Speed curves of input shaft and output shaft

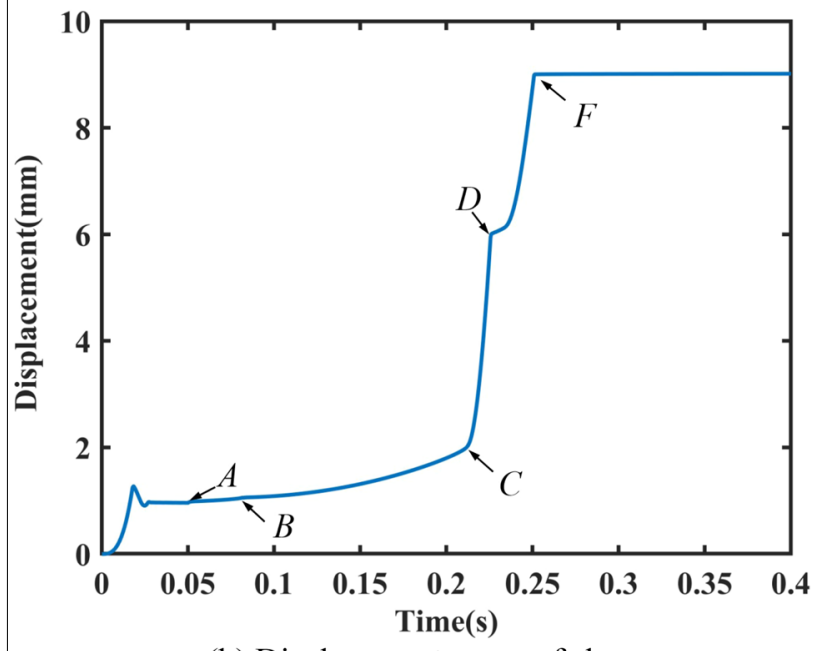

(b) Displacement curve of sleeve

Figure 2 Transmission movement state during shifting

and then enter the synchronization phase $A B$. The speed difference is eliminated at point $B$ to complete synchronization. After the synchronization is completed, the synchro ring is turned in the $B C$ section. At this time,

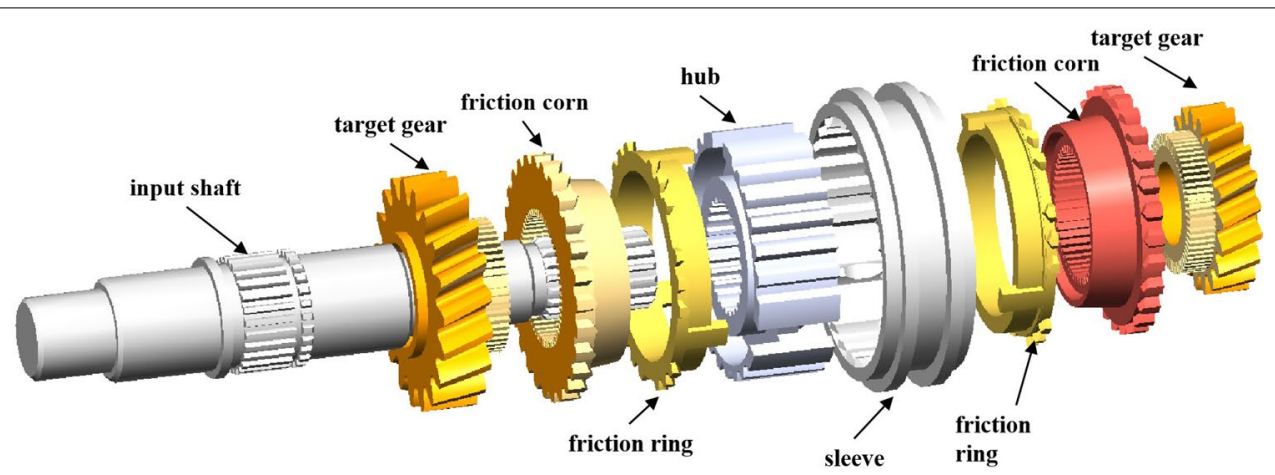

Figure 1 The structure and assembly relationship of ILRS 
the synchro ring and the target gear rotate at the same speed, and the turn-ring phase is completed at point $C$. The sleeve continues to move to contact the target gear at point $D$ and turns the target gear teeth. Point $E$ is the collision between the teeth of sleeve and the teeth of the target gear. $F$ point completes the movement of the sleeve to realize gear shifting. It can be seen from the $B E F$ stage that the speed difference produced by the ILRS during the turn-teeth phase will not disappear, and can only disappear through the collision between the teeth.

\subsection{Shifting Impact of ILRS}

Impact is an important indicator for evaluating shifting quality. It is expressed as Eq. (1).

$$
j=\frac{\mathrm{d}^{2} v}{3.6 \mathrm{~d} t^{2}}=\frac{\mathrm{d} a}{\mathrm{~d} t}=\frac{\mathrm{d} \alpha}{\mathrm{d} t} R_{w}=\frac{\mathrm{d} T_{w}}{I_{\nu} \mathrm{d} t} R_{w},
$$

where $v$ is the longitudinal speed of vehicle. $a$ represents the longitudinal velocity of the vehicle. $\alpha$ is the wheel angular acceleration. $T_{w}$ is the wheel driving torque. $I_{v}$ is the moment of inertia of the wheel. $R_{w}$ is the wheel radius.

The impact degree is derived from the change in the longitudinal speed of the vehicle [26]. The software realizes the shifting simulation with a certain simulation step length, so it is not exactly the same as the actual situation. The second derivation of the curve will make the deviation larger. The degree of impact can also be calculated by derivation of the wheel torque. At the moment when the sleeve teeth contacts the internal teeth of the friction cone ring, the impact torque is also generated by the teeth surface collision and the generation time is very short, which can reflect the impact characteristics, so it is also meaningful to measure the shifting impact. In addition, the impact torque only needs to derive the longitudinal velocity once, and the resulting error will be smaller.

The torque is expressed as follows:

$$
T_{w}=\frac{I_{v} \mathrm{~d} v}{R_{w} \mathrm{~d} t} .
$$

Using the impact torque instead of impact to evaluate the shifting quality, the torque is expressed as follows:

$$
T_{w}=I_{v} \cdot \alpha=I_{v} \cdot \frac{\alpha_{2}}{i_{0}}
$$

where $\alpha_{2}$ is the angular acceleration of input shaft. $i_{0}$ is the gear ratio of the transmission.

The shifting simulation with different shifting forces and different shifting modes is carried out to reflect the mechanical characteristics of the ILRS. The PID position control is used to directly realize the shifting, and different $P$ values are used to realize different shifting forces, as shown in Figure 3, when the shifting force decreases, the shifting time is longer, and the shifting impact torque decreases. It can be seen from Figure 3 that in several sets of the shifting force $(F)$, when the $F$ value is $170 \mathrm{~N}$, the synchronization time is $0.0813 \mathrm{~s}$, which is close to the synchronization time of the PCFRS in Section 5, so the $F$ value for the second shifting mode is $170 \mathrm{~N}$.

The second shifting strategy is that when the speed difference between the sleeve and the target gear is eliminated, the shifting force value achieved by the smaller gear shifting force. The simulation results are shown in Figure 4 . The smaller the gear shifting force, the smaller the speed difference is produced by the shifting, the longer the shifting time will be. This shifting strategy will produce a smaller impact torque compared to the pure position control. In order to compare with the PCFRS simulation results in Section 5, a group that produces

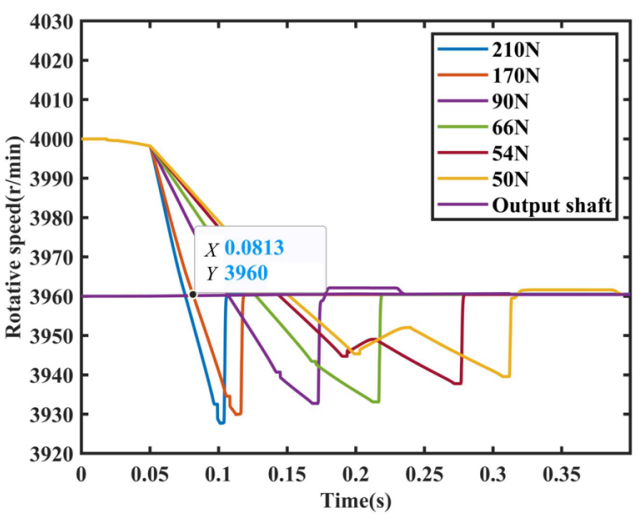

(a) The speed relationship between input shaft and output shaft

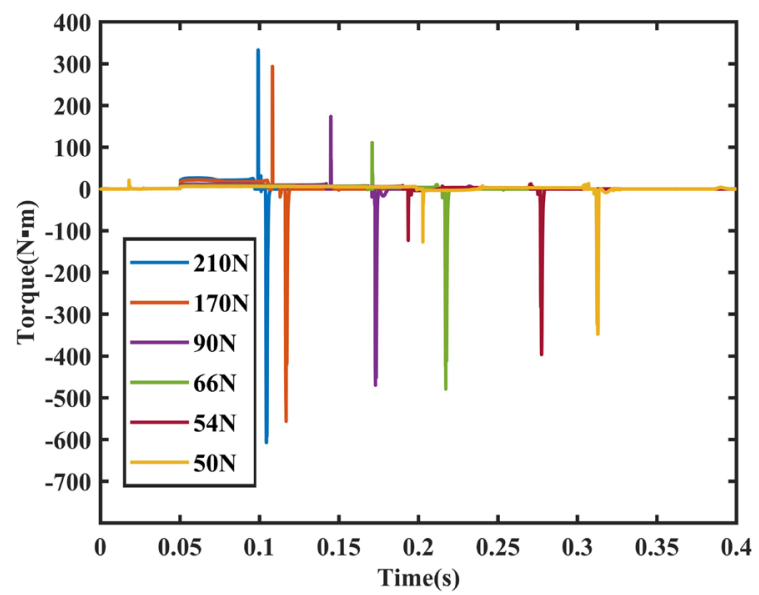

(b) Impact torque curves of different shifting modes

Figure 3 Shifting effect achieved by ILRS with single position control 




(a) The speed relationship between input shaft and output shaft

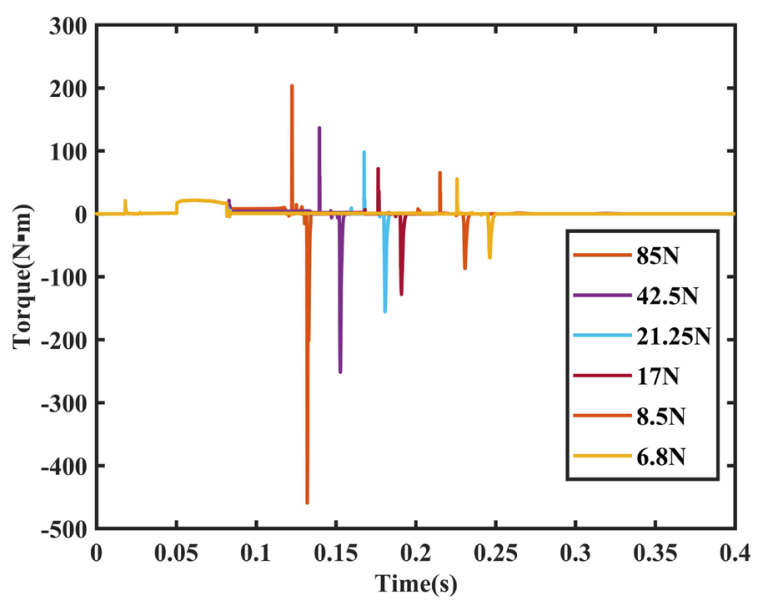

(b) Impact torque curves of different shifting modes

Figure 4 Shifting effect of ILRS adopts different shifting force

impact torque is found, and the time required to shifting is $0.2486 \mathrm{~s}$ when the impulsive torque is $69.8256 \mathrm{~N} \cdot \mathrm{m}$.

\subsection{Sliding Power Work with ILRS Shifting}

The sliding friction work is mainly expressed in the form of heat, which directly affects the reliability of the synchronizer. It may even cause irreversible damage to the synchronizer and affect its lifespan. This section will verify whether the shifting mode meets the requirements. According to the definition of sliding work, sliding work $L_{f}$ can be expressed as follows:

$$
L_{f}=\int_{0}^{\theta} T_{f} \mathrm{~d} \theta .
$$

From Eq. (4), $T_{f}$ is:

$$
T_{f}=\frac{F_{a} f R_{m}}{\sin \beta},
$$

where $F_{a}$ is the axial contact force of the friction cone ring and the synchro ring. $f$ is the friction coefficient of friction cone and synchro ring friction pair. $\theta$ is the relative rotation angle of the friction cone ring relative to the friction cone surface of the target gear during the synchronous friction process, $\mathrm{d} \theta=\left|\omega_{g}-\omega_{r}\right| \mathrm{d} t . \beta$ is the angle of friction cone. The analysis of the sliding work is shown as Eq. (6) [27]:

$$
L_{f}=\int_{0}^{t_{T}} \frac{\pi F_{a} f R_{m}}{30 \sin \beta}\left|\omega_{g}-\omega_{r}\right| \mathrm{d} t,
$$

where $t_{T}$ is the relative sliding time of friction cone ring and synchro ring. $\omega_{g}, \omega_{r}$ are the rotation speed of the target gear and the synchro ring.

In order to make the evaluation more objective, the specific sliding work and specific sliding power are introduced to evaluate the performance.

The specific sliding work is expressed as follows:

$$
q=\frac{L_{f}}{A_{f}},
$$

where $A_{f}$ is the contact area of the friction cone and the synchro ring.

In the synchronization stage, the instantaneous specific sliding power is used. The calculation can be calculated by dividing the contact area by the product of the friction torque and the relative angular velocity difference, which is expressed as follows:

$$
q_{p}=\frac{\pi T_{f} \cdot\left|\omega_{g}-\omega_{r}\right|}{30 A_{f}} .
$$

The simulation of different strategies for the ILRS fully demonstrates its mechanical characteristics. As shown in Figures 5 and 6, they are the specific sliding work and specific sliding power generated during the shifting simulation process of the two shifting strategies. The specific sliding work and specific sliding power are far less than the allowable value $0.09 \mathrm{~J} / \mathrm{mm}^{2}$ and $0.45 \mathrm{~W} / \mathrm{mm}^{2}$ [27]. It shows that the shifting simulation results of the ILRS meet the requirements of the mechanism, and the simulation data is reasonable.

\subsection{PV Value Analysis with ILRS Shifting}

Regarding the working conditions of the synchronizer, the $P V$ value is often used to check [28], that is, the product value of the surface pressure of the friction cone and the relative sliding speed. The $P V$ value can intuitively reflect whether the shifting force and speed difference set during the shifting process are reasonable. 


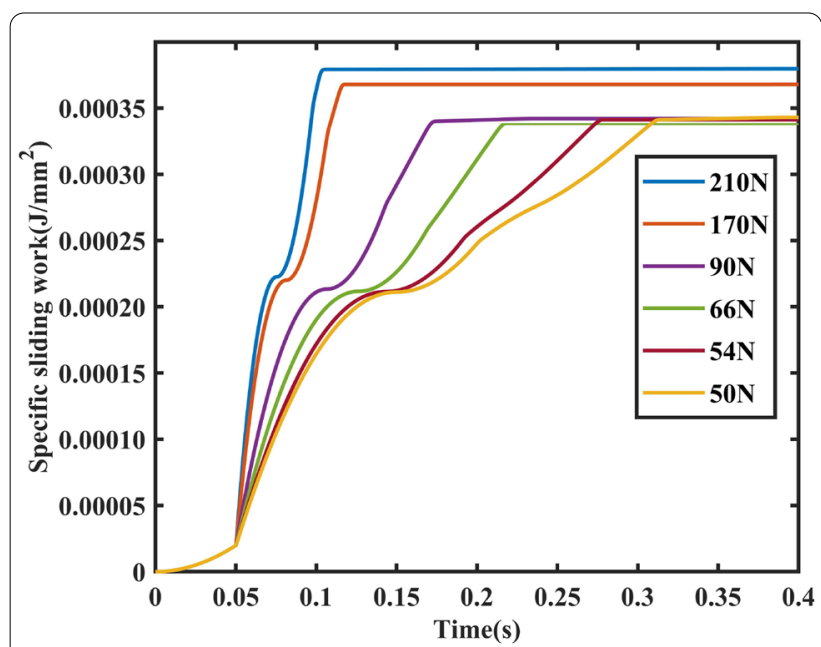

(a) Specific sliding work curves

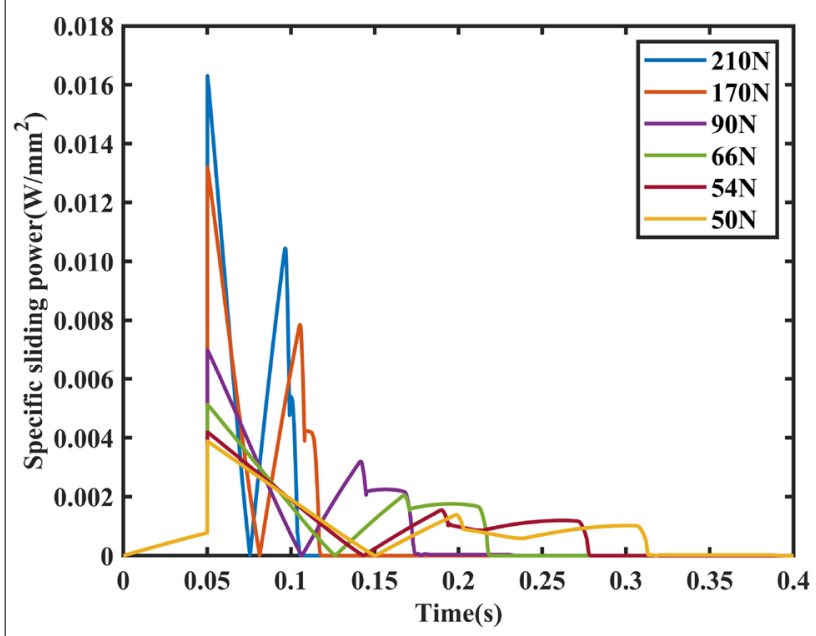

(b) Specific sliding power curves

Figure 5 Using different shifting forces to directly realize position-controlled shifting

The surface pressure $P$ of the $P V$ value is expressed as:

$$
P=\frac{F_{a}}{\sin \beta \cdot A_{f}} .
$$

The relative sliding speed $V$ of the $P V$ value is expressed as:

$$
V=\frac{100 \pi \cdot R_{m} \cdot\left|\omega_{g}-\omega_{r}\right|}{3} .
$$

Organizing Eqs. (9) and (10) get Eq. (11):

$$
P V=\frac{100 F_{a} \cdot \pi \cdot R_{m} \cdot\left|\omega_{g}-\omega_{r}\right|}{3 \sin \alpha \cdot A_{f}}<[P][V] .
$$

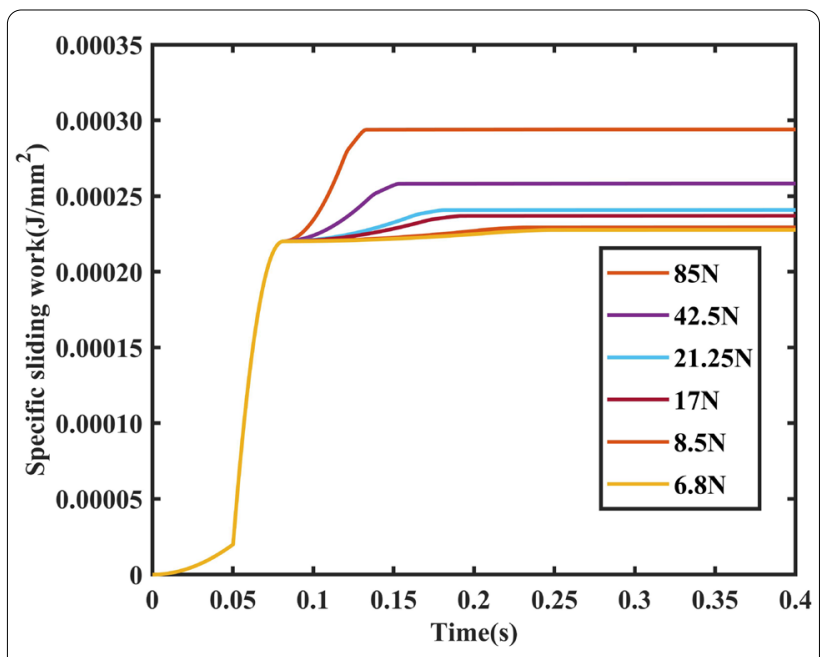

(a) Specific sliding work curves

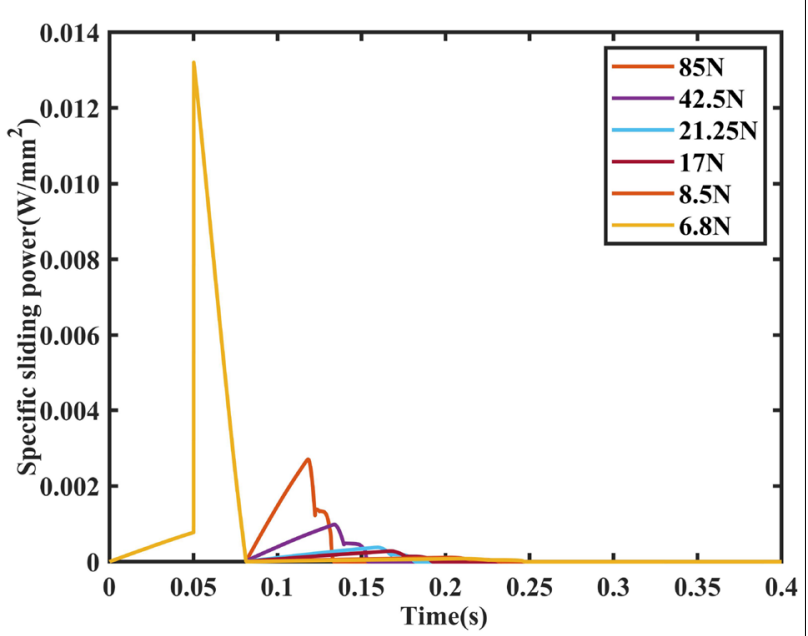

(b) Specific sliding power curves

Figure 6 Using different shifting forces after synchronization to achieve position-controlled shifting

According to ZF Friedrichshafen AG's recommendation, the allowable pressure in the contact area of the friction cone is $3 \mathrm{~N} / \mathrm{mm}^{2}$, and the allowable relative sliding speed is $5000 \mathrm{~mm} / \mathrm{s}$. So, the $P V$ value should not be greater than $15,000 \mathrm{~N} /(\mathrm{mm} \cdot \mathrm{s})$ [27].

The $P V$ values corresponding to the two shifting strategies are shown in Figure 7, the simulated $P V$ values are far less than the allowable value of $15,000 \mathrm{~N} /$ $(\mathrm{mm} \cdot \mathrm{s})$. Therefore, the shifting simulation results meet the working conditions of the synchronizer.

\subsection{Experiment Analysis of ILRS Shifting}

To verify the actual use effect of ILRS, a pure electric vehicle is adopted which equipped with a new type of 


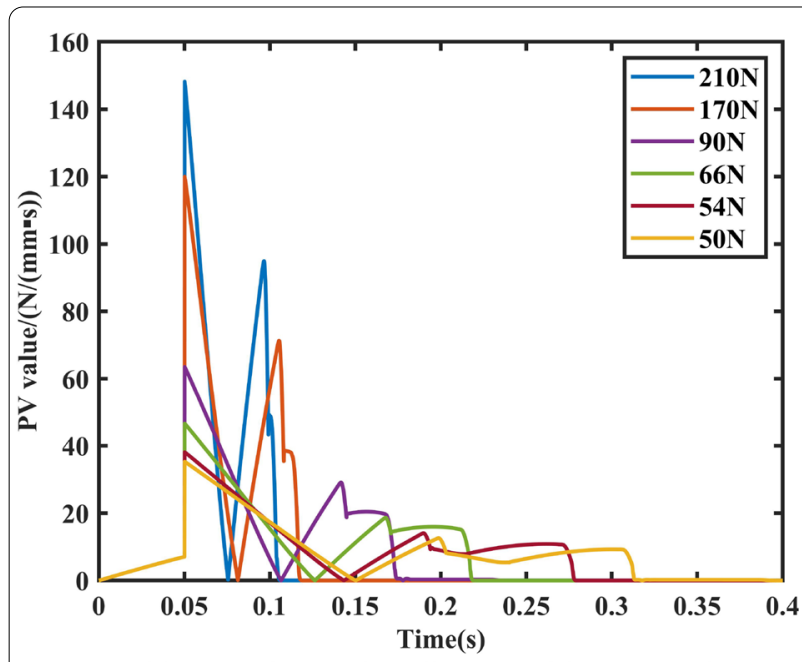

(a) $P V$ values using direct position control

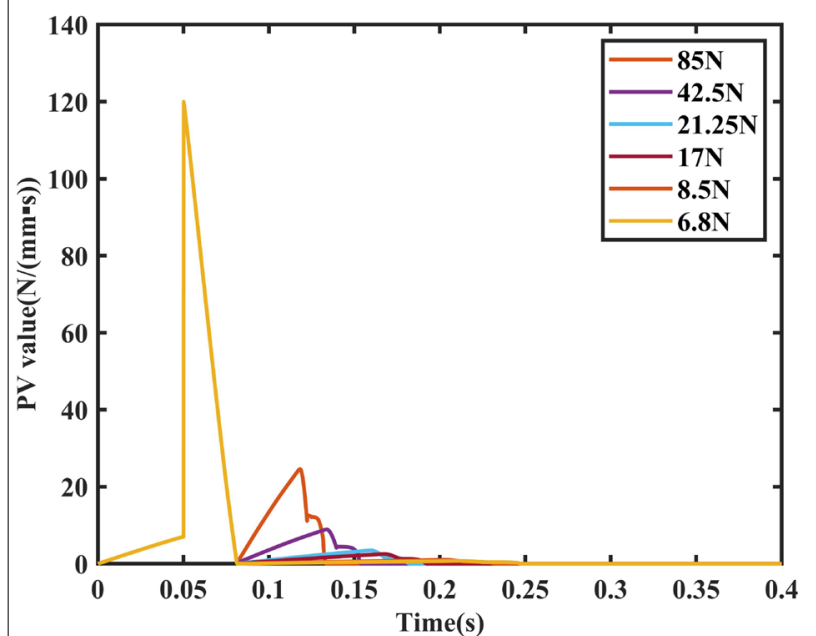

(b) $P V$ values for position control with different shifting forces after synchronization

Figure 7 Synchronizer shifting $P V$ value for different shifting modes

Clutchless AMT and ILRS is selected as the shift actuator, they are shown in Figure 8.

In order to reflect the working characteristics of the ILRS more intuitively, the experiment adopts a similar way to the simulation. For the sake of safety, the test shift speed is lower than the simulation shift speed. The test curves are shown in Figure 9. In the figure, the blue curve is the displacement of the sleeve. The speed of the input shaft and the target gear connected to the output shaft will fluctuate at the shifting time. At first, the sleeve is in the middle position, that is, the transmission is in neutral state. Then the input shaft speed decreases rapidly in the synchronization stage. In the stage of turn-teeth, the speed of input shaft decreases



(a) Test vehicle

(b) The new Clutchless AMT

Figure 8 Test vehicle and transmission

and the speed of output shaft increases. The experimental results are consistent with the simulation results. The speed difference produced by the shifting cannot be reduced or eliminated by the mechanism and will inevitably cause impact. Due to the gap between the teeth surfaces, the teeth surfaces will not contact quickly after completing the tooth entry. So in order to better complete the shifting, the speed of the motor connected to the input shaft is gradually increased to reduce the speed difference when the sleeve advance is completed.

The analysis is based on the structural characteristics of ILRS. If the motor speed is not adjusted when the sleeve advance is completed, the speed difference generated by the tooth can only be eliminated by collision. In addition, as can be seen from the figure, although the speed of motor is adjusted, the speed of the output shaft will fluctuate. Therefore, according to the experiment, the simulation data is consistent with the actual phenomenon. The speed difference produced by turning teeth is not self-elimination will produce an impact. Motor speed regulation requires high precision and the speed of the

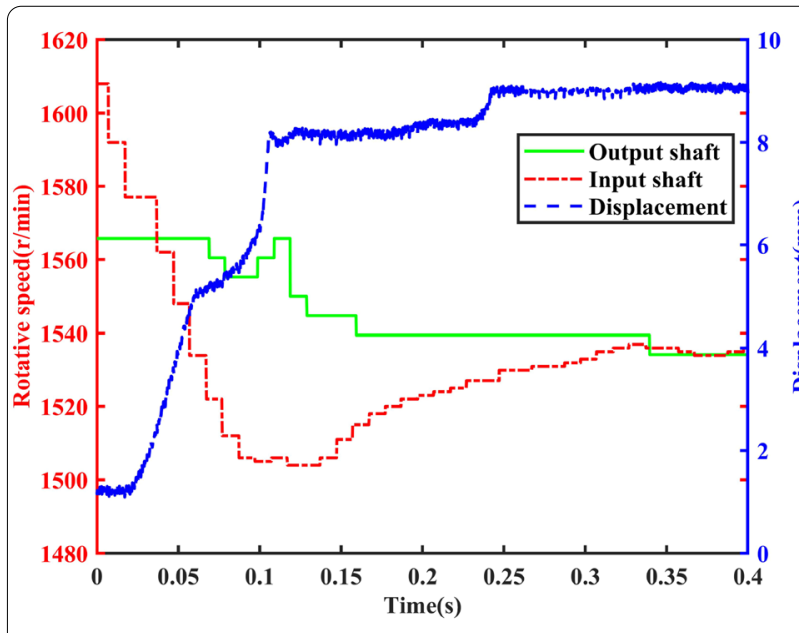

Figure 9 The speed of the shaft and the displacement of the sleeve 
input shaft also fluctuates to the speed of the output shaft when adjusting speed. In a word, ILRS has inevitable drawbacks.

\section{Working Mechanism Analysis of the PCFRS}

Based on the problem presented by ILRS, the PCFRS is designed. The structure of PCFRS is shown in Figure 10, which includes hub, sleeve, wave spring, synchro ring, friction cone ring. The synchro ring and the sleeve are used to eliminate the speed difference and engage the hub with the target gear. The hub is connected to the input shaft for combining the sleeve and the synchro ring. The shifting force is transmitted by the spring between the sleeve and the synchro ring. After the synchronization is completed, the sleeve directly compresses the wave ring close to the teeth to realize the turn-teeth and complete the shifting. If the rotational speed of the motor is directly adjusted enter the speed range of the gear advancement, the sleeve is directly controlled to complete the shifting. If the rotational speed does not reach the gear-advancing speed range, the sleeve will move a certain distance and wait until the gear-advancing speed range is synchronously reached before completing the gear shifting to ensure the reliability of gear shifting.

The functions of PCFRS are as follows:

The hub is fixed on the input shaft, which is connected to the drive motor, forces the synchro ring and the sleeve to rotate at the same speed.

The sleeve is moved axially on the hub, connecting the target gear and the input shaft to achieve driving.

The synchro ring is the friction unit. It rubs the friction cone ring fixed on the target gear to realize the synchronization of the input shaft and target gear speed.

The two sides of the wave spring touch the sleeve and the synchro ring, respectively. During shifting, the sleeve is moved axially and pushing the wave spring touch the synchro ring. The synchro ring is touching with the friction cone ring under the action of spring compression. The wave spring is further compressed to complete the gear shifting.
The friction cone ring is fixed on the target gear, the outer side rub the synchro ring to synchronize the speed, and the end surface is opened with internal teeth holes to guide the sleeve teeth to complete the gear shifting.

Compared with the ILRS, the PCFRS has the following advantages:

(1) There is no pre-synchronization and no need to squeeze the slider with excess force during the shifting process, which makes the shifting process control more stable and reliable.

(2) There is no lock teeth and turn-ring phase, and there is no need to worry about the inability to synchronize the speed due to the failure of the lock teeth of the synchro ring, which saves shifting time, does not need to produce two shifting impacts and makes the synchronizer work more reliable.

(3) The sleeve can move before the speed difference is completely eliminated in the synchronization phase, and it can be set to move when the speed difference is in the allowable range, which make shifting more efficient.

(4) The working characteristics are related with the speed regulation characteristics of the motor, and the process is flexible and effective. According to different speed differences, it can adopt direct shifting or shifting after synchronization to the corresponding speed difference, which is more suitable for the shifting of pure electric vehicle transmissions.

(5) Synchronous friction torque always exists during the shifting process, so that the speed difference generated after turn-teeth is quickly reduced, and impact-free shifting can be realized, which making shifting easier to control.

(6) There are fewer components than ILRS and no sliding block mechanism and sudden shifting force.

The limitation of the design is that the friction is still needed to synchronize firstly and then to shifting. The

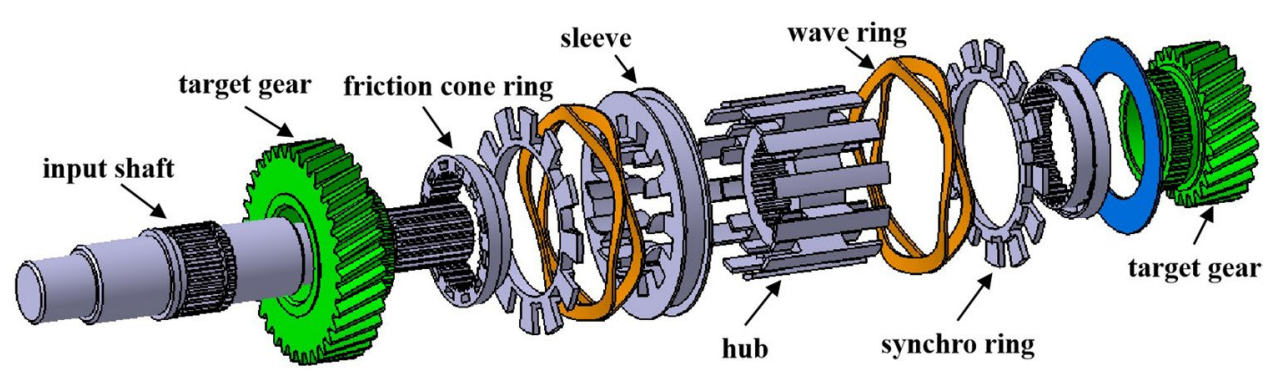

Figure 10 The structure and assembly relationship of PCFRS 
friction will bring decrease the lifespan of friction elements. However, according to relevant literature studies, pure electric vehicles are suitable for two-speed transmissions [29-31]. The low-gear transmission of pure electric vehicles does not shifting frequently, thus reducing the drawbacks of friction loss in shifting. In order to highlight the mechanical characteristics of the PCFRS, this paper will compare the shifting characteristics of the PCFRS and the ILRS. In order to verify that the PCFRS adopts wave springs to meet the working requirements during the entire shifting process, this paper will verify the working conditions of each shifting. In addition, the shifting actuator will always bear the pressure brought by the wave spring. The problem can be solved by adopting a worm gear shifting actuator. According to the self-locking characteristics of the worm gear, the synchronizer can help the vehicle shifting easily. The simple shifting control strategy of the pressure controllable friction ring synchronizer is as follows:

(1) The sleeve moves a certain distance, and then the wave spring is compressed to make the synchro ring rub the friction cone ring for synchronization;

(2) When the speed difference is enter the required range, the sleeve will continue to move at a certain speed;

(3) After the sleeve touches the internal teeth of the friction cone ring, the contact force of the teeth surface causes a certain speed difference, and then the latter two are separated;

(4) The sleeve continues to move at a certain speed, and the friction element eliminates the speed difference at a faster speed while the sleeve moves, and finally realizes a zero-speed-difference shifting.

\section{Phased Dynamics Modeling \\ of the Synchronization Process}

The model of the transmission system with a PCFRS is shown in Figure 11, where s represents the sleeve, $r$ represents the synchro ring, and g represents the target gear. $I_{1}$ is the moment of inertia of the motor. $I_{g}$ is the moment of inertia of the target gear. $I_{x}, I_{e}, I_{f}$ are the moment of inertia of the meshing gear, $I_{v}$ is the moment of inertia of the wheel. $T_{1}, T_{v}$ are drive torque of the motor and wheel torque. $c_{i}$ is the damping coefficient of the corresponding component, where $i=0,1,2,3,4 . k_{i}$ is the stiffness coefficient of the corresponding component, where $i=0$, 2. The PCFRS is mounted on the input shaft. This section will combine the transmission system to analyze the dynamics characteristics of the PCFRS.

In order to illustrate the analysis of the shifting process of the PCFRS, the sleeve, the wave spring, the

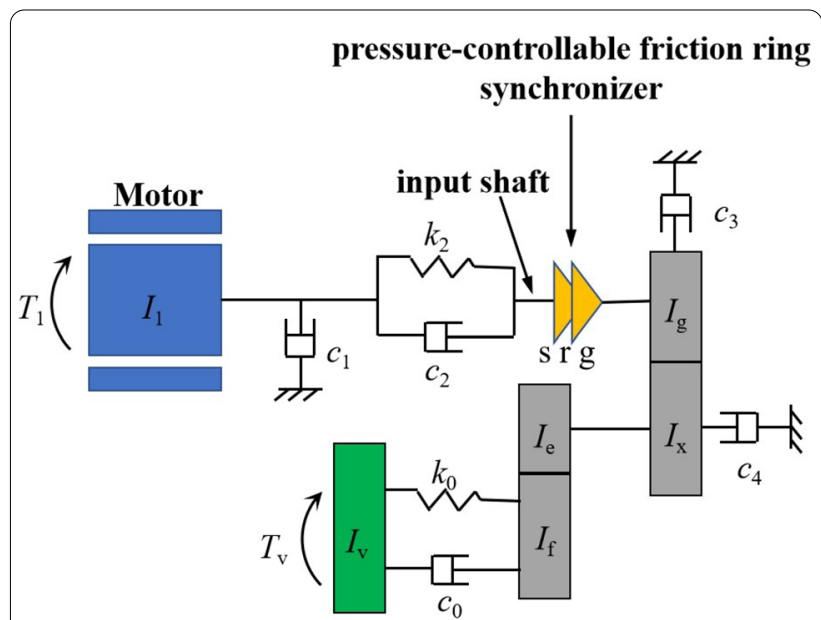

Figure 11 The transmission system with PCFRS

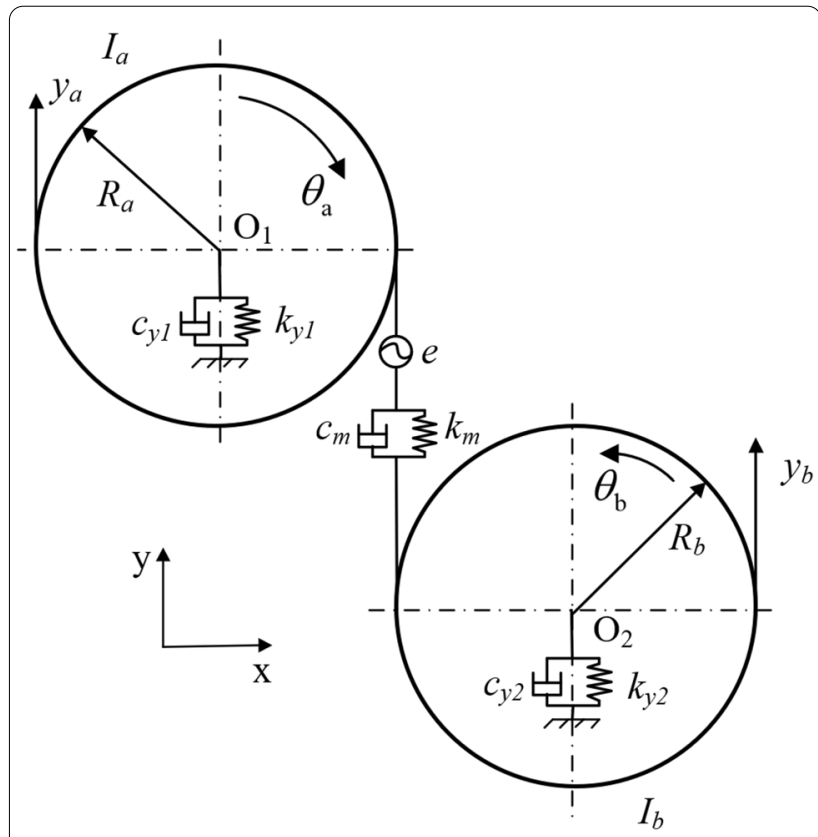

Figure 12 Non-linear dynamics model of gear meshing

synchro ring and the friction cone ring are used to articulate the working mechanism. The whole process of the shifting of the sleeve is divided into multiple stages. In order to express the dynamics relationship of the gear meshing process more clearly, the gear contact model is adopted [32], as shown in Figure 12. Where $R_{a}$ and $R_{b}$ are the basic radius of the gear, $\theta_{a}$ and $\theta_{b}$ are the torsional freedom of the driving gear and the driven gear, $c_{y 1}, c_{y 2}, k_{y 1}$ and $k_{y 2}$ are the supporting damping coefficient and supporting stiffness of the driving gear and the driven gear, respectively. $c_{m}$ and $k_{m}$ are the 
damping coefficient and stiffness of the driving gear and driven gear meshing. $I_{a}, I_{b}$ are the moment of inertia of the meshing gear. $e$ is the static transmission error of the gear pair.

The relative displacement of the driving gear and the driven gear due to torsion in the normal coordinate system is

$$
y_{0}=R_{a} \theta_{a}-R_{b} \theta_{b} .
$$

In fact, the process of synchronously eliminating the speed difference and the timing of shifting are both random. This section selects the most representative stages that can cover all situations. Combined with the dynamics model establishment method proposed [33], it is expressed as follows.

Stage 1: The sleeve moves to make the synchro ring contact the friction cone ring.
The state of PCFRS in the first stage is shown in Figure 13. At this stage, the sleeve is in the middle position of the synchronizer, and the wave spring is in the free length state. The sleeve moves axially toward the target gear under the guiding action of the hub, pushing the wave spring to contact the synchro ring. The spring force pushes the synchro ring to move axially toward the target gear under the guidance of the hub and the synchro ring quickly contact the friction cone surface on the outside of the friction cone ring. When the sleeve continues to move and reaches the set position, the wave spring gets a certain degree of compression and the shifting process is in stage 2 or stage 3 .

$$
\begin{aligned}
& I_{1} \ddot{\theta}_{1}=T_{1}-c_{1} \dot{\theta}_{1}-c_{2}\left(\dot{\theta}_{1}-\dot{\theta}_{s}\right)-k_{2}\left(\theta_{1}-\theta_{s}\right), \\
& \left(I_{s}+I_{r}\right) \ddot{\theta}_{s}=c_{2}\left(\dot{\theta}_{1}-\dot{\theta}_{s}\right)+k_{2}\left(\theta_{1}-\theta_{s}\right),
\end{aligned}
$$

$$
I_{f} \ddot{\theta}_{f}=R_{f}\left[\begin{array}{c}
c_{m}\left(\dot{y}_{02}+\dot{y}_{e}-\dot{y}_{f}-\dot{e}_{y 2}\right) \\
+k_{m}\left(y_{02}+y_{e}-y_{f}-e_{y 2}\right)
\end{array}\right]-c_{0}\left(\dot{\theta}_{f}-\dot{\theta}_{v}\right)-k_{0}\left(\theta_{f}-\theta_{v}\right),
$$

$$
I_{v} \ddot{\theta}_{v}=c_{0}\left(\dot{\theta}_{f}-\dot{\theta}_{v}\right)+k_{0}\left(\theta_{f}-\theta_{v}\right)-T_{v}
$$

where $I_{s}$ is the moment of inertia of the hub and the sleeve. $I_{r}$ is the moment of inertia of the synchro ring. $\theta_{i}$ is the rotation angle of the corresponding component, $\dot{\theta}_{i}$ is the angular velocity of the corresponding component, $\ddot{\theta}_{i}$ is the angular acceleration of the corresponding component, where $i=\mathrm{s}, \mathrm{g}, \mathrm{x}, \mathrm{f}, \mathrm{v}, 1 . R_{j}$ is the basic radius of the corresponding gear, $y_{j}$ are the translational displacement of the corresponding gear. $\dot{y}_{j}$ are the translational velocity of the corresponding gear, where $j=\mathrm{g}, \mathrm{x}, \mathrm{e}, \mathrm{f} . y_{01}$ and $y_{02}$ are the relative displacement of meshing gear. $\dot{y}$ ${ }_{01}$ and $\dot{y}_{02}$ are the relative velocity of meshing gear. $e_{y 1}, e_{y 2}$ are the static transmission error of the gear pair. $\dot{e}_{y 1}, \dot{e}_{y 2}$ are the static transmission velocity error of the gear pair. $F_{\text {shift }}$ is the force of shifting.

Stage 2: Synchro ring and friction cone ring contact for synchronization.

The state of PCFRS at this stage is shown in Figure 14. The sleeve stops moving in the axial direction, and the spring force generated by the spring compression makes the friction cone surface of the synchro ring and the fric-

tion cone ring keep in touch. The friction surface will be
Figure 13 Schematic diagram of the structure of the sleeve in the middle position of the synchronizer 

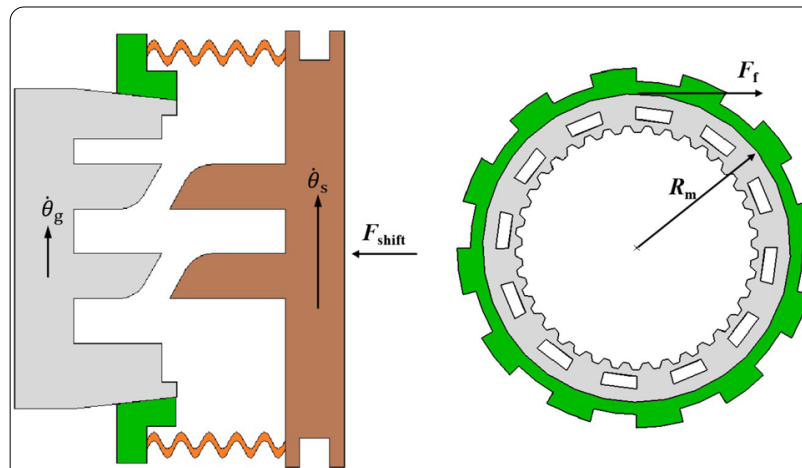

Figure 14 Schematic diagram of the synchronization process of the synchro ring and the friction cone ring contacting

produced friction torque, because there is a speed difference between the synchro ring and the friction cone ring. The synchro ring is constrained by the hub, so that the rotational speed of the PCFRS and the input shaft gradually become consistent. At this stage, the shifting motor stops driving, the stop of the sleeve is realized by the self-locking characteristic of the worm gear. When the speed difference is reached the allowable teeth-entering range by the friction torque, this stage ends and the shifting process will proceed to stage 3 .

$$
\begin{aligned}
& I_{1} \ddot{\theta}_{1}=T_{1}-c_{1} \dot{\theta}_{1}-c_{2}\left(\dot{\theta}_{1}-\dot{\theta}_{s}\right)-k_{2}\left(\theta_{1}-\theta_{s}\right) \\
& \left(I_{s}+I_{r}\right) \ddot{\theta}_{s}=c_{2}\left(\dot{\theta}_{1}-\dot{\theta}_{s}\right)+k_{2}\left(\theta_{1}-\theta_{s}\right)-F_{f} R_{m}, \\
& I_{g} \ddot{\theta}_{g}=F_{f} R_{m}-c_{3} \dot{\theta}_{g}-R_{g}\left[c_{m}\left(\dot{y}_{01}+\dot{y}_{g}-\dot{y}_{x}-\dot{e}_{y 1}\right)+k_{m}\left(y_{01}+y_{g}-y_{x}-e_{y 1}\right)\right], \\
& \left(I_{x}+I_{e}\right) \ddot{\theta}_{x}=-c_{4} \dot{\theta}_{x}+R_{x}\left[\begin{array}{c}
c_{m}\left(\dot{y}_{01}+\dot{y}_{g}-\dot{y}_{x}-\dot{e}_{y 1}\right) \\
+k_{m}\left(y_{01}+y_{g}-y_{x}-e_{y 1}\right)
\end{array}\right]-R_{e}\left[\begin{array}{c}
c_{m}\left(\dot{y}_{02}+\dot{y}_{e}-\dot{y}_{f}-\dot{e}_{y 2}\right) \\
+k_{m}\left(y_{02}+y_{e}-y_{f}-e_{y 2}\right)
\end{array}\right], \\
& I_{f} \ddot{\theta}_{f}=R_{f}\left[\begin{array}{c}
c_{m}\left(\dot{y}_{02}+\dot{y}_{e}-\dot{y}_{f}-\dot{e}_{y 2}\right) \\
+k_{m}\left(y_{02}+y_{e}-y_{f}-e_{y 2}\right)
\end{array}\right]-c_{0}\left(\dot{\theta}_{f}-\dot{\theta}_{v}\right)-k_{0}\left(\theta_{f}-\theta_{v}\right),
\end{aligned}
$$



Figure 15 Schematic diagram of the structure of the sleeve and the friction cone ring contacting

the sleeve can move again without completely eliminating the speed difference. When the speed difference reaches the allowable range, the sleeve moves to contact the internal teeth of the friction cone ring to turn teeth, and the contact between the sleeve and the friction cone ring generates surface contact force. At this time, there is a friction torque between the friction cone ring and the

$$
I_{\nu} \ddot{\theta}_{v}=c_{0}\left(\dot{\theta}_{f}-\dot{\theta}_{v}\right)+k_{0}\left(\theta_{f}-\theta_{v}\right)-T_{v},
$$

where $F_{f}$ is the friction force between the synchro ring and the friction cone ring. $R_{m}$ is the friction equivalent radius.

Stage 3: Before the speed difference is eliminated, the sleeve enters the teeth and the friction cone ring teeth contact.

The state of PCFRS at this stage is shown in Figure 15. According to the shifting characteristics of the PCFRS, synchro ring. There is also a turn-teeth torque between the friction cone ring and the sleeve. Both friction torque and turn-teeth torque promote the elimination of the speed difference. Under the action of the helical teeth, the sleeve moves to the internal teeth hole of the friction cone ring. At the end of this phase, the shifting process proceeds to stage 4 or stage 5 .

$$
I_{1} \ddot{\theta}_{1}=T_{1}-c_{1} \dot{\theta}_{1}-c_{2}\left(\dot{\theta}_{1}-\dot{\theta}_{s}\right)-k_{2}\left(\theta_{1}-\theta_{s}\right),
$$




$$
\begin{aligned}
& \left(I_{s}+I_{r}\right) \ddot{\theta}_{s}=c_{2}\left(\dot{\theta}_{1}-\dot{\theta}_{s}\right)+k_{2}\left(\theta_{1}-\theta_{s}\right)-F_{f} R_{m}-F_{t} R_{t}, \quad \begin{array}{l}
\text { the sleeve has not been fully meshed. Therefore, stage } \\
(26)
\end{array} \quad 4 \text { is required. The sleeve continues to turn the friction } \\
& I_{g} \ddot{\theta}_{g}=F_{t} R_{t}+F_{f} R_{m}-c_{3} \dot{\theta}_{g}-R_{g}\left[c_{m}\left(\dot{y}_{01}+\dot{y}_{g}-\dot{y}_{x}-\dot{e}_{y 1}\right)+k_{m}\left(y_{01}+y_{g}-y_{x}-e_{y 1}\right)\right] \\
& \left(I_{x}+I_{e}\right) \ddot{\theta}_{x}=-c_{4} \dot{\theta}_{x}+R_{x}\left[\begin{array}{c}
c_{m}\left(\dot{y}_{01}+\dot{y}_{g}-\dot{y}_{x}-\dot{e}_{y 1}\right) \\
+k_{m}\left(y_{01}+y_{g}-y_{x}-e_{y 1}\right)
\end{array}\right]-R_{e}\left[\begin{array}{c}
c_{m}\left(\dot{y}_{02}+\dot{y}_{e}-\dot{y}_{f}-\dot{e}_{y 2}\right) \\
+k_{m}\left(y_{02}+y_{e}-y_{f}-e_{y 2}\right)
\end{array}\right] \\
& I_{f} \ddot{\theta}_{f}=R_{f}\left[\begin{array}{c}
c_{m}\left(\dot{y}_{02}+\dot{y}_{e}-\dot{y}_{f}-\dot{e}_{y 2}\right) \\
+k_{m}\left(y_{02}+y_{e}-y_{f}-e_{y 2}\right)
\end{array}\right]-c_{0}\left(\dot{\theta}_{f}-\dot{\theta}_{v}\right)-k_{0}\left(\theta_{f}-\theta_{v}\right),
\end{aligned}
$$

$$
I_{\nu} \ddot{\theta}_{v}=c_{0}\left(\dot{\theta}_{f}-\dot{\theta}_{v}\right)+k_{0}\left(\theta_{f}-\theta_{v}\right)-T_{v},
$$

where $F_{t}$ is the tangential component of the turn-teeth force. $F_{N}$ is the positive pressure on the bevel. $R_{t}$ is the turn-teeth radius. $\gamma$ is the teeth surface angle.

Stage 4: After the speed is eliminated, the sleeve teeth moves in contact with the friction cone ring teeth.

The state of PCFRS at this stage is shown in Figure 16. This situation exists when the speed difference between the sleeve and the friction cone ring is eliminated, but

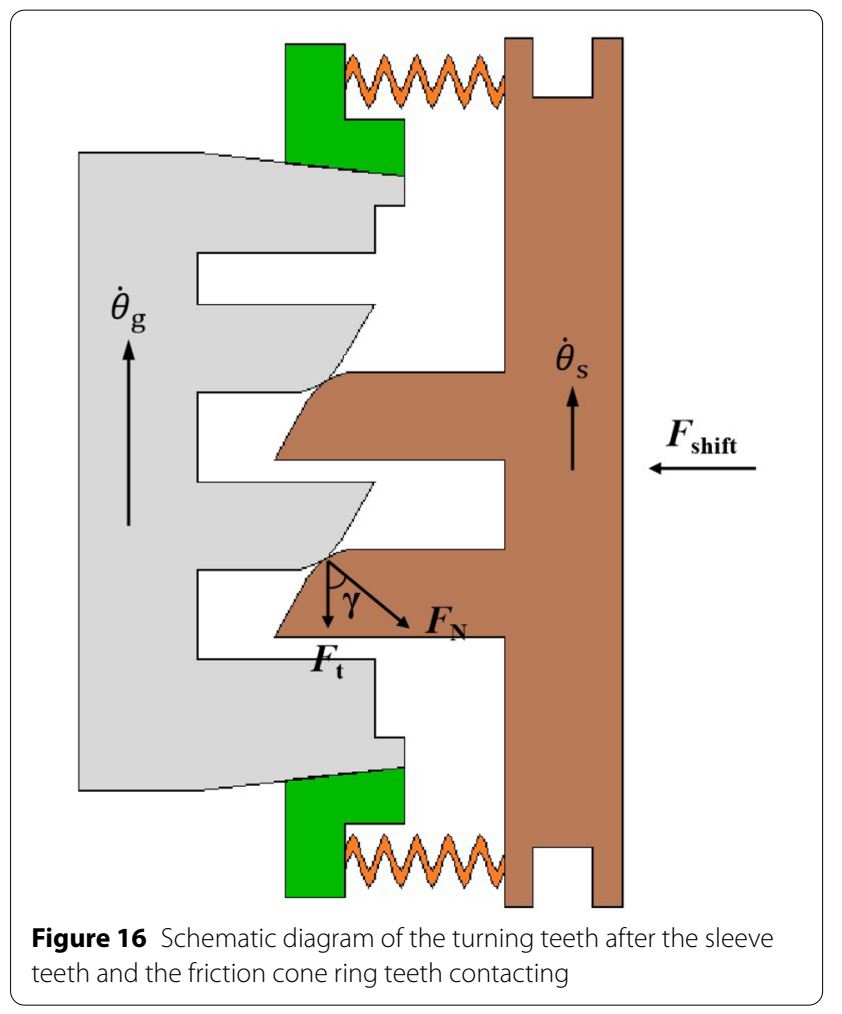

cone ring teeth and advance the teeth. The gear shifting method at this stage is that the sleeve teeth are slowly advanced so that the teeth surface is in continuous contact, so that no excessive great speed difference is generated, and finally a successful meshing without a speed difference is achieved.

$$
I_{1} \ddot{\theta}_{1}=T_{1}-c_{1} \dot{\theta}_{1}-c_{2}\left(\dot{\theta}_{1}-\dot{\theta}_{s}\right)-k_{2}\left(\theta_{1}-\theta_{s}\right)
$$

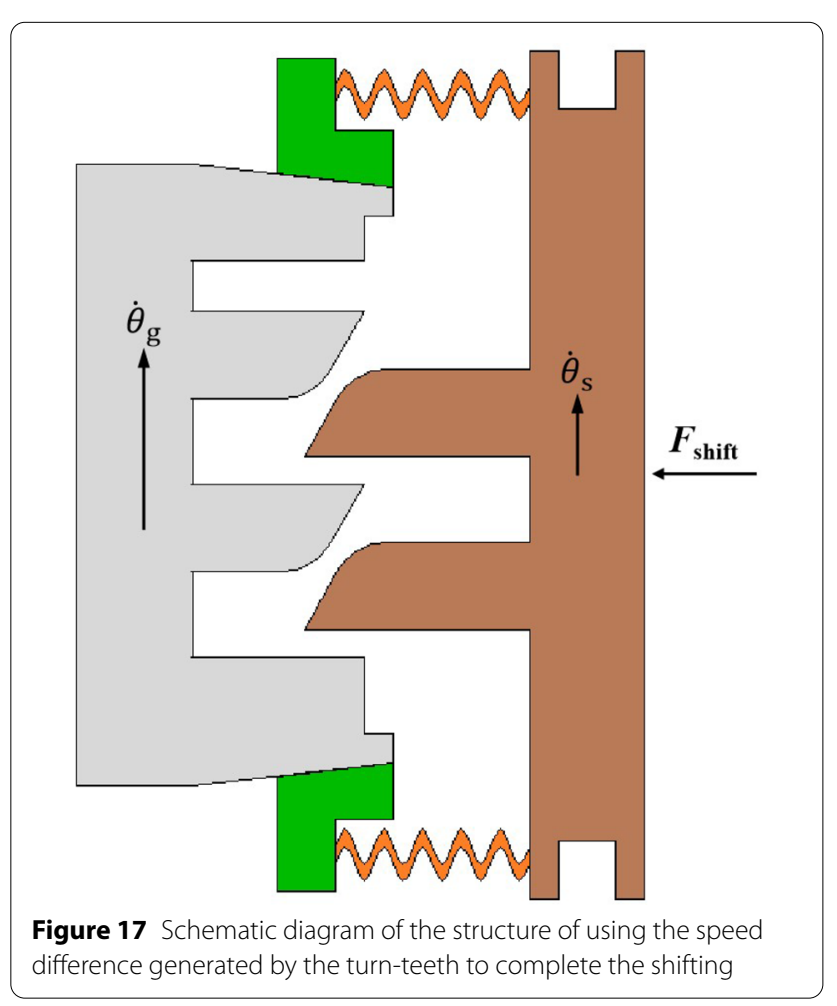




$$
\begin{aligned}
& \left(I_{s}+I_{r}\right) \ddot{\theta}_{s}=c_{2}\left(\dot{\theta}_{1}-\dot{\theta}_{s}\right)+k_{2}\left(\theta_{1}-\theta_{s}\right)+F_{f} R_{m}-F_{t} R_{t}, \quad \begin{array}{l}
\text { the speed difference, and then } \\
\text { ment is used. The difference }
\end{array} \\
& I_{g} \ddot{\theta}_{g}=F_{t} R_{t}-F_{f} R_{m}-c_{3} \dot{\theta}_{g}-R_{g}\left[c_{m}\left(\dot{y}_{01}+\dot{y}_{g}-\dot{y}_{x}-\dot{e}_{y 1}\right)+k_{m}\left(y_{01}+y_{g}-y_{x}-e_{y 1}\right)\right], \\
& \left(I_{x}+I_{e}\right) \ddot{\theta}_{x}=-c_{4} \dot{\theta}_{x}+R_{x}\left[\begin{array}{c}
c_{m}\left(\dot{y}_{01}+\dot{y}_{g}-\dot{y}_{x}-\dot{e}_{y 1}\right) \\
+k_{m}\left(y_{01}+y_{g}-y_{x}-e_{y 1}\right)
\end{array}\right]-R_{e}\left[\begin{array}{c}
c_{m}\left(\dot{y}_{02}+\dot{y}_{e}-\dot{y}_{f}-\dot{e}_{y 2}\right) \\
+k_{m}\left(y_{02}+y_{e}-y_{f}-e_{y 2}\right)
\end{array}\right], \\
& I_{f} \ddot{\theta}_{f}=R_{f}\left[\begin{array}{c}
c_{m}\left(\dot{y}_{02}+\dot{y}_{e}-\dot{y}_{f}-\dot{e}_{y 2}\right) \\
+k_{m}\left(y_{02}+y_{e}-y_{f}-e_{y 2}\right)
\end{array}\right]-c_{0}\left(\dot{\theta}_{f}-\dot{\theta}_{v}\right)-k_{0}\left(\theta_{f}-\theta_{v}\right),
\end{aligned}
$$

the speed difference, and then the slow teeth advancement is used. The difference in speed reduces the

$$
I_{\nu} \ddot{\theta}_{v}=c_{0}\left(\dot{\theta}_{f}-\dot{\theta}_{v}\right)+k_{0}\left(\theta_{f}-\theta_{v}\right)-T_{\nu},
$$

Stage 5: After the speed is eliminated, the contact between the sleeve and the teeth surface of the friction cone produces a speed difference. The generated speed difference is used to complete the teeth advancement.

The state of PCFRS at this stage is shown in Figure 17. It exists in the situation of stage 4, but it is not the slow gear advancement. The teeth advancement according to the teeth advancement state of the stage 3 , resulting in relative angle between the sleeve and the teeth hole of the friction cone ring to realize shifting. At this stage, there may be impact during meshing, but the difference in speed is small, the impact produced is within an acceptable range. In addition, the speed difference can also be eliminated when the teeth is advanced to realize the speed difference-free shifting.

$$
\begin{aligned}
& I_{1} \ddot{\theta}_{1}=T_{1}-c_{1} \dot{\theta}_{1}-c_{2}\left(\dot{\theta}_{1}-\dot{\theta}_{s}\right)-k_{2}\left(\theta_{1}-\theta_{s}\right), \\
& \left(I_{s}+I_{r}\right) \ddot{\theta}_{s}=c_{2}\left(\dot{\theta}_{1}-\dot{\theta}_{s}\right)+k_{2}\left(\theta_{1}-\theta_{s}\right)+F_{f} R_{m},
\end{aligned}
$$

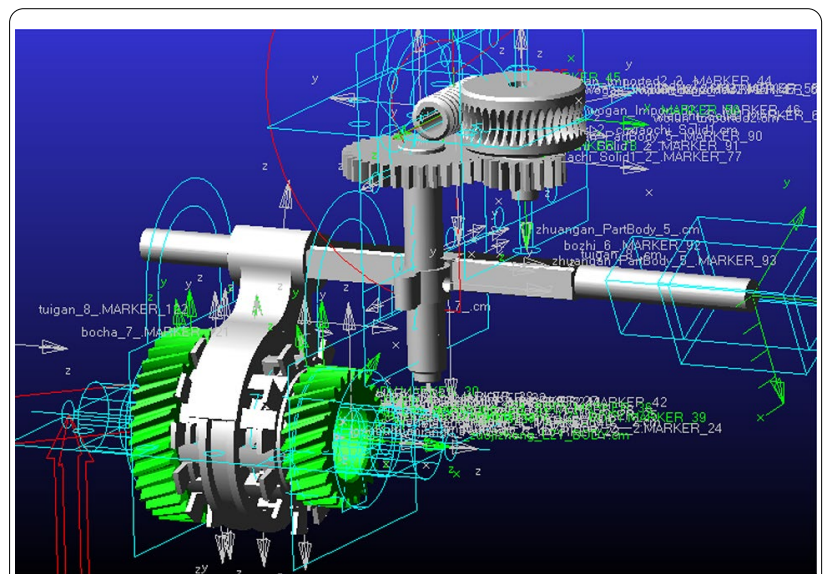

Figure 18 Multi-body dynamics modeling based on ADAMS
Table 1 Main parameters of the synchronizers

\begin{tabular}{ll}
\hline Symbol & Value \\
\hline$I_{\mathrm{v}}\left(\mathrm{kg} \mathrm{m}^{2}\right)$ & 78 \\
$R_{\mathrm{w}}(\mathrm{m})$ & 0.266 \\
$R_{\mathrm{m}}(\mathrm{m})$ & 0.025 \\
$i_{0}$ & 5.2542 \\
$F$ & 0.11 \\
$B^{\left({ }^{\circ}\right)}$ & 6.5 \\
$A_{\mathrm{f}}\left(\mathrm{mm}^{2}\right)$ & 628.319 \\
\hline
\end{tabular}




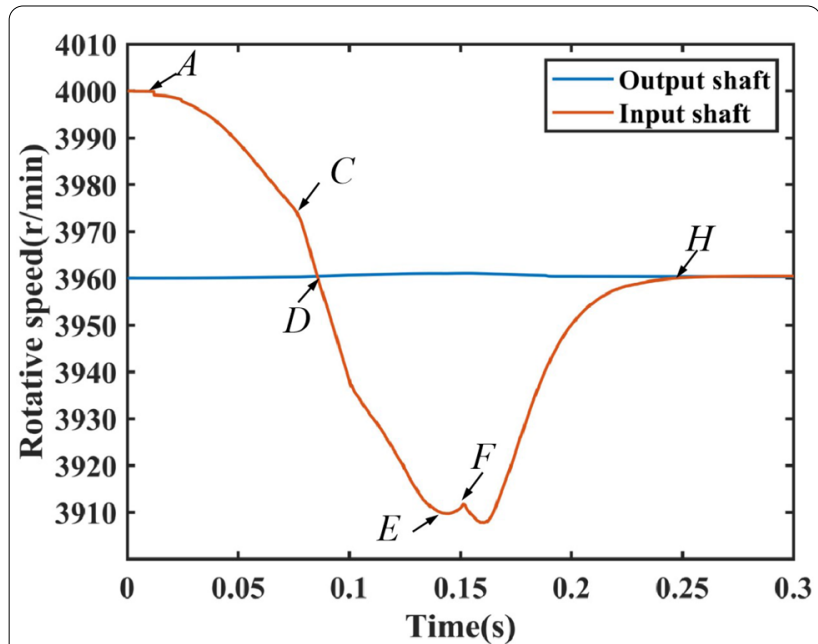

(a) Speed curves of input shaft and output shaft

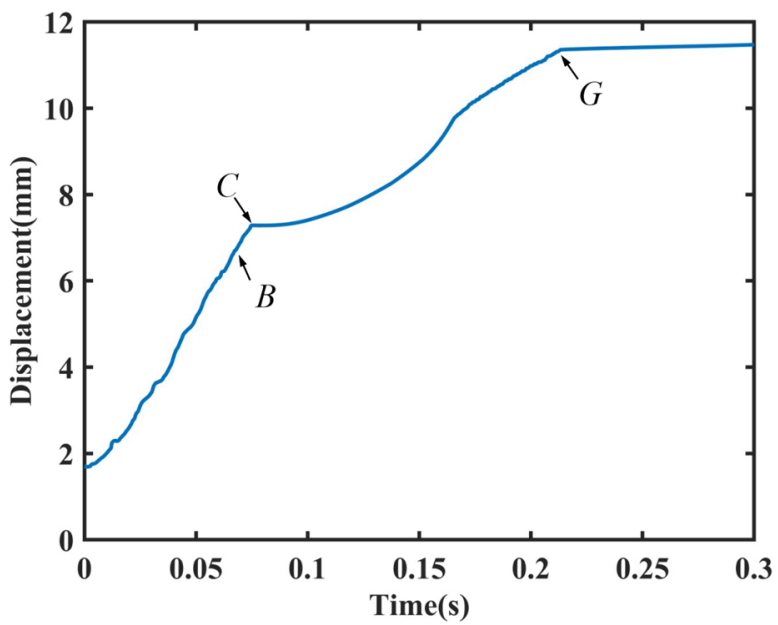

(b) Displacement curve of sleeve

Figure 19 Transmission movement state during shifting

$$
I_{\nu} \ddot{\theta}_{v}=c_{0}\left(\dot{\theta}_{f}-\dot{\theta}_{v}\right)+k_{0}\left(\theta_{f}-\theta_{v}\right)-T_{v},
$$

The relative angle of the friction cone ring teeth hole is also random, and there may be a small or zero relative angle to the friction cone ring teeth hole during the teeth engagement process, so the shifting is completed directly after the gear is dialed or the gear is directly engaged.

\section{Simulation Analysis of the PCFRS and Comparison with ILRS Simulation Results}

In this paper, ADAMS is used to establish to perform the multi-body dynamics modeling and debugging. The model is shown in Figure 18. In order to simplify the simulation process, the target gear replaces the gear meshing system in the transmission as the output shaft, converts the wheel inertia to the target gear, and sets the specified initial speed and moment of inertia for the target gear, the



(a) The relationship between input shafts

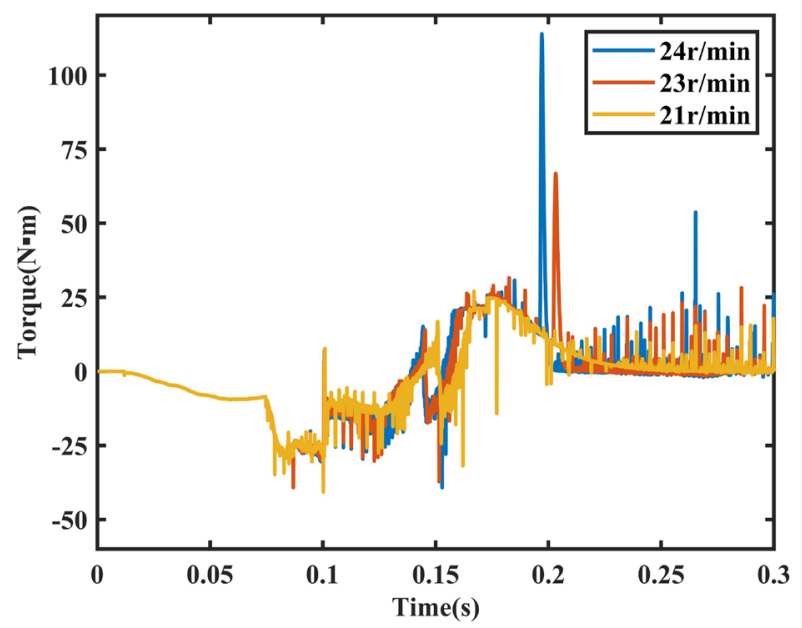

(b) Impact torque curves of different shifting effects and target gear output speed

Figure 20 Shifting effect of PCFRS shifting at different moving speeds of the sleeve

input shaft and the synchronizer. After that, the ADAMS and MATLAB/Simulink co-simulation was used to realize the shifting simulation. Combining the structural characteristics of the PCFRS, firstly, the PID position controller is used to move the sleeve to the specified position, and after synchronization is completed, another PID speed controller is used to control the shifting motor to realize shifting.

PCFRS simulation adopts the same parameters and conditions as ILRS simulation. Table 1 shows the main parameters of the simulation of the PCFRS and the ILRS.

\subsection{Simulation Analysis of the PCFRS Shifting}

The shifting process of PCFRS is shown in Figure 19, which shows the relationship between the rotational 
speed of the sleeve and the output shaft at all stages of the shifting.

Combining the rotational speed curves of the input shaft and the displacement curve of the sleeve, point $A$ in the figure is that the gap between the components of the shifting process is eliminated and the idle stroke of the sleeve moving in the direction of the target gear is completed. As the movement of the sleeve enters the synchronization phase, point $B$ indicates that the sleeve to reach the set position, and then the speed difference is determined. When the speed reaches the range of sleeve advancement, the sleeve advancement continues. Point $C$ indicates that the sleeve is in contact with the friction cone ring teeth. Then it enters the turnteeth phase and the teeth surface contact in the gear

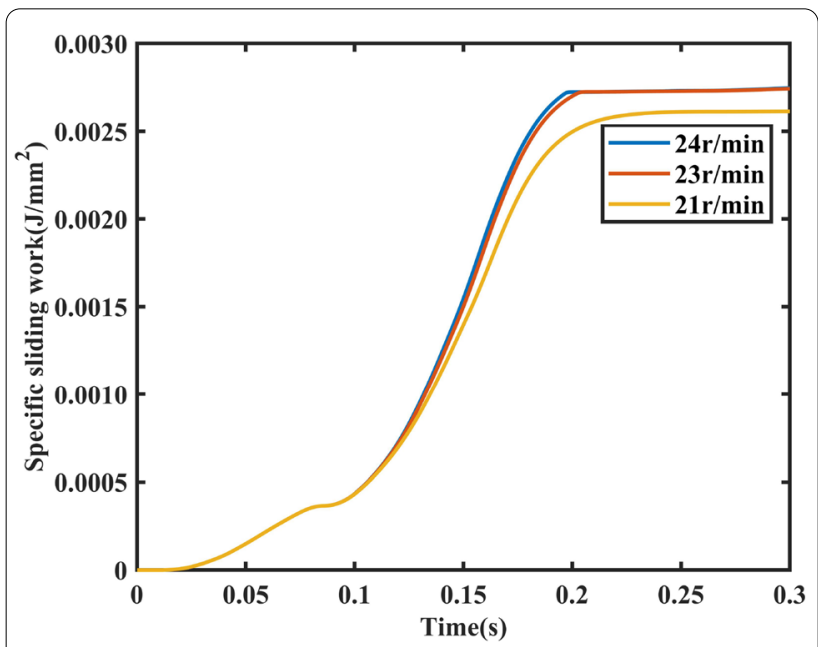

(a) Specific sliding work curves



(b) Specific sliding power curves

Figure 21 The specific sliding work and specific sliding power of the PCFRS under different shifting speeds shifting stage produces a force and the friction cone ring to accelerate the synchronization process. The synchronization is completed at point $D$, at the same time the sleeve is moved at a certain speed. At point $E$, the sleeve teeth are separated from the friction cone ring teeth, and the friction torque continues to be synchronized while the engagement sleeve is moving. At point $F$, the sleeve teeth contacts the friction cone ring gear again, and after the contact continues to turn teeth, which produces a speed difference and the teeth separate. The sleeve teeth still advances at a certain speed, and the friction torque quickly eliminates the speed difference. At point $G$, the advancement of the sleeve is completed, and there is still a small speed difference at this time. Due to the small gap between the sleeve teeth and the teeth groove of the friction cone ring, synchronization is still performed after the advancement of sleeve is completed. At point $H$, the speed difference is completely eliminated, achieving no speed difference shifting.

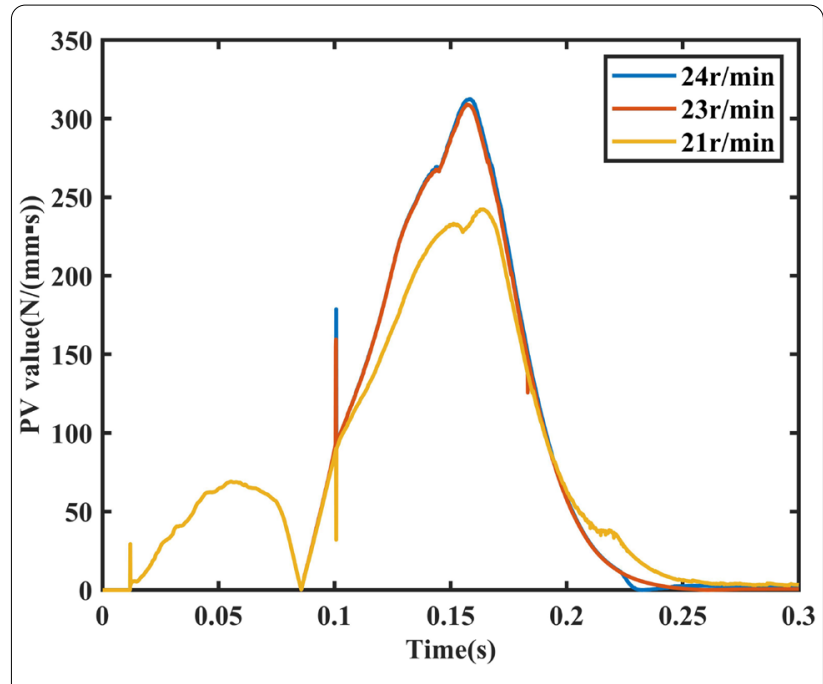

Figure $22 P V$ value curves of PCFRS

Table 2 Simulation data comparison between ILRS and PCFRS

\begin{tabular}{llll}
\hline $\begin{array}{l}\text { Synchronizer } \\
\text { type }\end{array}$ & $\begin{array}{l}\text { Synchronization } \\
\text { time (s) }\end{array}$ & $\begin{array}{l}\text { Impact } \\
\text { torque } \\
\text { (N. } \mathbf{m})\end{array}$ & Shifting time (s) \\
\hline ILRS & 0.0813 & 69.8256 & 0.2486 \\
PCFRS & 0.0856 & 66.5673 & 0.2031 \\
& & non & 0.2406 \\
\hline
\end{tabular}




\subsection{Shifting Quality Analysis}

\subsubsection{Shifting Impact Analysis}

As shown in Figure 20, different shifting effects with different moving speeds of the sleeve are adopted, and it takes a long time to achieve a shifting without a speed difference of $0.2406 \mathrm{~s}$, but the impact torque of teeth collision is not generated. When the shifting produces the impact torque of $66.5673 \mathrm{~N} \cdot \mathrm{m}$ in the shifting mode, the shifting time is only $0.2031 \mathrm{~s}$. The result will be compared with the simulation results of the ILRS in Section 2.

\subsubsection{Sliding Power Analysis}

This section will verify whether the shifting process meets the standard through the sliding work to further determine the rationality of the PCFRS shifting simulation, which makes the comparison between the PCFRS and the ILRS more convincing. Figure 21 shows the specific sliding work and specific sliding power between the friction elements during the shifting process.

The allowable values of specific sliding work and specific sliding power are $0.09 \mathrm{~J} / \mathrm{mm}^{2}$ and $0.45 \mathrm{~W} / \mathrm{mm}^{2}$ [27]. It can be seen from the figures that the specific sliding work and specific sliding power of the PCFRS in different shifting modes meet the allowable value.

\subsubsection{PV Value Analysis}

The simulation curves are shown in Figure 22. When the sleeve moves at different speeds, the working conditions of the synchronizer all meet the allowable value and meet the requirements of use. The simulation of the above shifting modes is reasonable.

\subsection{Comparison between PCFRS and ILRS Shifting}

This paper aims to highlight the advantages of the PCFRS from the aspect of mechanical characteristics by comparing the PCFRS and the ILRS. If there is a speed difference to realize the shifting, the impact torque will be generated. In Table 2, the shifting data of the ILRS and the PCFRS with similar impact torque are selected, and the data of the PCFRS without speed difference shifting data is selected for comparison and explanation.

The ILRS cannot eliminate the speed difference generated during turn-teeth phase, and it will eventually generate impact torque. In order to produce a smaller impact torque, a smaller shifting force must be adopted at the expense of shifting time. It can be seen from the table that although the ILRS takes $0.0813 \mathrm{~s}$ to complete the synchronization, when it produces an impact torque similar to that of the PCFRS, the shifting time is longer and the impact torque is still greater. The shifting quality of PCFRS is much better than that of ILRS.

\section{Conclusions}

As a universal synchronizer in AMT, ILRS is not suitable for pure electric vehicles without a clutch. The simulation and test researches in this paper prove the conclusion. ILRS has very high requirements on the speed regulation and response time of the motor. To ensure the quality of shifting, the motor must be able to achieve accurate and rapid speed adjustment. In addition, even if the performance of the motor can be guaranteed, the tooth profile of the synchronizer itself will cause a secondary impact on the tooth, which is unavoidable. The above factors will reduce the shifting quality of ILRS.

PCFRS is suitable for clutchless AMTs of pure electric vehicle, which is verified by mechanical characteristics and work rationality simulation analysis. The research results show that the impact torque can eliminate the speed difference in the turn-teeth phase to achieve no speed difference shifting. Through the verification of evaluation indexes such as specific sliding work and $P V$ value model, it is concluded that the shifting process of PCFRS meets the allowable value.

The shifting simulation results of the PCFRS and the ILRS are compared. The wave spring can act on the PCFRS during the shifting process to make the synchronous friction torque always work. Moreover, the PCFRS does not have the turn-ring phase and the lockteeth phase, which makes the time for shifting without speed difference to be as short as $0.2406 \mathrm{~s}$. When the PCFRS adopts the mode of shifting with a smaller speed difference, the required shifting time is shorter to $0.2031 \mathrm{~s}$. Compared with the ILRS that shifting time is $0.2486 \mathrm{~s}$, the impact torque is smaller as well as the time required is shorter.

In addition, the mechanism studied in this paper still need to be proved by bench or actual vehicle loading test. Affected by the epidemic, the production of PCFRS has not been completed, the next step will be to test and verify. Besides, the structure of the PCFRS has been determined, but the corresponding size is only preliminary determined in combination with the structural characteristics. In the next step, the specific size will be further optimized.

\section{Acknowledgements \\ Not applicable.}

\section{Authors' Contributions}

LZ was in charge of the conceptualization, methodology, formal analysis and funding acquisition; YP wrote manuscripts and used software for research and data processing. HY reviewed and edited the article. SL verified the content of the article, supervised it, and obtained funds. All authors read and approved the final manuscript. 


\begin{abstract}
Authors' Information
Lipeng Zhang, born in 1979, is currently a professor at Yanshan University, China. He received his PhD degree from Beijing Institute of Technology, China, in 2011. His research interests include intelligent vehicle dynamics and control, new energy vehicle compound transmission, driver awareness and driving assistance.

Yunao Peng, born in 1996, is currently a master candidate at Hebei Key Laboratory of Special Delivery Equipment, Yanshan University, China.

Haojie Yang, born in 1998, is currently a master candidate at Hebei Key Laboratory of Special Delivery Equipment, Yanshan University, China.

Shaohua Li, born in 1973, is currently a professor at State Key Laboratory of Mechanical Behavior and System Safety of Traffic Engineering Structures, Shijiazhuang Tiedao University, Shijiazhuang 050043, China. Her research interest is vehicle dynamics and control.
\end{abstract}

\section{Funding}

Supported by National Natural Science Foundation of China (Grant No. 51775478), Natural Science Foundation of Hebei Province (Grant Nos. E2020203078, E2020203174), Open Project of State Key Laboratory of Mechanical Behavior and System Safety of Traffic Engineering Structures (Grant No. KF2021-11) and Graduate Innovation Funding Project of Hebei Province (Grant No. CXZZSS2021063).

\section{Competing Interests}

The authors declare no competing financial interests.

\section{Author Details}

${ }^{1}$ Hebei Key Laboratory of Special Delivery Equipment, Yanshan University, Qinhuangdao 066004, China. ${ }^{2}$ State Key Laboratory of Mechanical Behavior and System Safety of Traffic Engineering Structures, Shijiazhuang Tiedao University, Shijiazhuang 050043, China.

Received: 27 April 2021 Revised: 23 December 2021 Accepted: 4 February 2022

Published online: 05 March 2022

\section{References}

[1] CTseng, CYu. Advanced shifting control of synchronizer mechanisms for clutchless automatic manual transmission in an electric vehicle. Mechanism and Machine Theory, 2015, 84: 37-56.

[2] P D Walker, S A Rahman, Z Bo, et al. Modelling, simulations, and optimisation of electric vehicles for analysis of transmission ratio selection. Advances in Mechanical Engineering, 2013, 5: 1-7.

[3] C H Yu, SY Goh. Study of seamless gear-shift strategy for a clutchless automated manual transmission, IEEE/SICE International Symposium on System Integration, Taipei, Taiwan, 2017: 11-14.

[4] H He, Z Liu, L Zhu, et al. Dynamic coordinated shifting control of automated mechanical transmissions without a clutch in a plug-in hybrid electric vehicle. Energies, 2012, 5(8): 3094-3109.

[5] X Xu, YT Luo. Modeling and analysis of gear shifting process of non-synchronizer AMT based on collision model. IEEE Access, 2021, 9: 13354-13367.

[6] YTian, N Zhang, S L Zhou, et al. Model and gear shifting control of a novel two-speed transmission for battery electric vehicles. Mechanism and Machine Theory, 2020, 152: 103902.

[7] CYu, CTseng. Research on gear-change control technology for the clutchless automatic-manual transmission of an electric vehicle. Proceedings of the Institution of Mechanical Engineers Part D Journal of Automobile Engineering, 2013, 227(10): 1446-1458.

[8] J Li, F Luo, Y Luo, et al. Sensitivity analysis on a synchronization mechanism for manual transmission gearbox. SAE Technical Papers, 2014, 1 1768.

[9] XY Wang, L Li, K He, et al. Position and force switching control for gear engagement of automated manual transmission gear-shift process. Journal of Dynamic Systems Measurement \& Control, 2018, 140(8): 4039184.

[10] W L Xu, W Zhao, B Su, et al. Investigation of manual transmission synchronizer failure mechanism induced by interface material/lubricant combinations. Wear, 2015, 3: 436-442.
[11] D T Qin, M Y Yao, S J Chen, et al. Shifting process control for two-speed automated mechanical transmission of pure electric vehicles. International Journal of Precision Engineering and Manufacturing, 2016, 17(5): 623-629.

[12] S S Lin, S Q Chang, B Li. Gearshift control system development for directdrive automated manual transmission based on a novel electromagnetic actuator. Mechatronics, 2014, 24(8): 1214-1222.

[13] L P Zhang, L Q Yang, X B Guo, et al. Stage-by-phase multivariable combination control for centralized and distributed drive modes switching of electric vehicles. Mechanism and Machine Theory, 2020, 147: 103752.

[14] Y Zhang, H Zhao, M M Qiu, et al. Model-based control of synchronizer shifting process for trajectory tracking control. International Journal of Automotive Technology, 2020, 21(4): 943-952.

[15] D Häggström, U Sellgren. The effect of manufacturing tolerances on the thermomechanical load of gearbox synchronizers. Procedia CIRP, 2018, 72 1202-1207.

[16] B Pang, J L Hong, B Z Gao, et al. Shift quality amelioration of EV with AMT by speed regulation. IFAC-Papers Online, 2018, 51(31): 910-917.

[17] M H Hu, L Chen, DY Wang, et al. Modeling and characteristic study of the shifting engagement process in stepped transmission. Mechanism and Machine Theory, 2020, 151: 103912.

[18] C H Wang, ZW Guo. Synchronizer multi-objective parameter optimization based on improved particle swarm algorithm. Journal of Mechanical Strength, 2019, 2: 356-362.

[19] ST Razzacki. Synchronizer design: a mathematical and dimensional treatise. SAE Transactions, 2004, 1: 1230.

[20] L Li, K He, X Wang, et al. Sensor fault-tolerant control for gear-shifting engaging process of automated manual transmission. Mechanical Systems \& Signal Processing, 2018, 99: 790-804.

[21] G Bóka, J Márialigeti, L Lovas, et al. Face dog clutch engagement at low mismatch speed. Periodica Polytechnica Transportation Engineering, 2010, 38(1): 29-35.

[22] C H Yu, CYTseng. Research on gear-change control technology for the clutchless automatic-manual transmission of an electric vehicle. Proceedings of the Institution of Mechanical Engineers, 2013, 227(10): 1446-1458.

[23] R Heath. Zeroshift's transmission technology. Atzautotechnology, 2008, 8: 44-49.

[24] W Mo, P D Walker, N Zhang. Dynamic analysis and control for an electric vehicle with harpoon-shift synchronizer. Mechanism and Machine Theory, 2019, 133: 750-766.

[25] W Mo, P Walker, Y Fang, et al. A novel shift control concept for multispeed electric vehicles. Mechanical Systems and Signal Processing, 2018, 112: 171-193.

[26] Z Z Lei, D Y Sun, Y G Liu, et al. Analysis and coordinated control of mode transition and shifting for a full hybrid electric vehicle based on dual clutch transmissions. Mechanism and Machine Theory, 2017, 114: 125-140.

[27] H Naunheimer. Automotive Transmission. Beijing: China Machine Press, 2011:233-282.

[28] L T Cao. Wear analysis and optimization design of synchronizer of two-speed transmission in pure electric vehicle. Hefei: Hefei University of Technology, 2016.

[29] M Mousavi, A Pakniyat, TWang, et al. Seamless dual brake transmission for electric vehicles: Design, control and experiment. Mechanism and Machine Theory, 2015, 94: 96-118.

[30] Y Tian, J Ruan, N Zhang, et al. Modelling and control of a novel two-speed transmission for electric vehicles. Mechanism and Machine Theory, 2018, 127: 13-32.

[31] B Z Gao, Q Liang, Y Xiang, et al. Gear ratio optimization and shift control of 2-speed I-AMT in electric vehicle. Mechanical Systems and Signal Processing, 2015, 50-51: 615-631.

[32] L P Zhang, D J Gu, B N Qi, et al. Variable-mode impact suppression method for electric vehicle dual-mode coupling drive system. Journal of Mechanical Engineering, 2018, 54(8): 165-176.(in Chinese)

[33] F K Omar, K A Moustafa, S Emam. Mathematical modeling of gearbox including defects with experimental verification. Journal of Vibration \& Control, 2012, 18(9): 1310-1321. 